Autor:

Dr. Gerd W. Zimmermann

Facharzt für Allgemeinmedizin

Kapellenstraße 9,

D-65719 Hofheim

\title{
Wird die diamorphingestützte Behandlung Heroinabhängiger zum Standard?
}

\begin{abstract}
- Im Gemeinsamen Bundesausschuss (G-BA) wird zurzeit die diamorphingestützte Behandlung Heroinabhängiger beraten. Hintergrund sind die gesetzlichen Änderungen im Betäubungsmittelgesetz und in der Betäubungsmittelverordnung (BtmVV) vom 15.7.2009, die eine diamorphingestützte Behandlung von Heroinabhängigen ermöglichen.

Die Kassenärztliche Bundesvereinigung (KBV) hatte sich seinerzeit gegen die gesetzliche Regelung ausgesprochen, die schließlich aber mit parlamentarischer Mehrheit doch beschlossen wurde. In der Begründung zu den gesetzlichen Änderungen wird ausgeführt, dass der G-BA nunmehr über die Übernahme dieser Diamorphinsubstitution in der GKV Regelungen treffen soll. Dort wurde mittlerweile mit einstimmiger Mehrheit eine schnelle Umsetzung eingeleitet.
\end{abstract}

MMW-Kommentar: Die Beratungen zur Diamorphinsubstitution im Zusammenhang mit den bereits bisher geltenden Substitutionsrichtlinien sind weit fortgeschritten und werden voraussichtlich spätestens Ende des 1. Quartals 2010 abgeschlossen sein. Sie laufen darauf hinaus, dass zukünftig - wie gesetzlich vorgesehen und bisher in den Modellpraxen erprobt - hierauf spezialisierte Ambulanzen unter bestimmten Bedingungen die Substitution mit Diamorphin zulasten der Gesetzlichen Krankenkassen durchführen dürfen. Hierzu benötigen sie die Genehmigung der zuständigen Kassenärztlichen Vereinigung (KV) und müssen bestimmte Vorgaben der Substitutionsrichtlinie (insbesondere Qualitätssicherungsmaßnahmen) erfüllen, wie sie bereits bisher für substituierende Praxen gelten.

\section{Rundfunkgebühren für den Internet-PC: alles weiterhin offen?}

\footnotetext{
- Mehrere Gerichte haben bisher entschieden, dass ein beruflich eingesetzter PC mit Internetzugang rundfunkgebührenpflichtig ist. Als Beispiel kann hier ein Urteil des Bayerischen Verwaltungsgerichtshofs vom 19.5.2009 angeführt werden (Az. 7 B 08.2922). Das Verwaltungsgericht Schleswig hat hingegen mit Urteil vom 2. Juli 2009 (Az. 14 A 243/08) festgestellt, dass für einen Personalcomputer (PC) keine Rundfunkgebühren zu zahlen sind, sofern eine Wiedergabe von Rundfunksendungen auf dem PC nicht möglich ist oder er nicht zum Empfang von Rundfunksendungen bereitgehalten wird.
}

Häusliches Arbeitszimmer

Doch wieder steuerbegünstigt?

- Nach einem Urteil des Finanzgerichts (FG) Köln vom 20. August

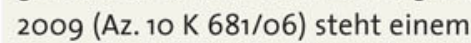
selbstständig tätigen Freiberufler mit einem Schreibtischarbeitsplatz in den Praxisräumen grundsätzlich kein anderer Arbeitsplatz im Sinne des $\$ 4$ Abs. 5 S. 1 des Einkommensteuergesetzes (EStG) zu. Das Urteil bezieht sich auf die bis einschließlich 2006 geltende Rechtslage, nach der Aufwendungen von bis zu 1250 Euro pro Jahr steuerlich geltend gemacht werden konnten, wenn die betriebliche oder berufliche Nutzung eines häuslichen Arbeitszimmers mehr als $50 \%$ der gesamten betrieblichen und beruflichen Tätigkeit beträgt oder kein anderer Arbeitsplatz zur Verfügung steht. Seit dem 1. Januar 2007 muss das Arbeitszimmer den Mittelpunkt der gesamten betrieblichen Betätigung darstellen.

MMW-Kommentar: Der Bundesfinanzhof hat verfassungsmäßige Zweifel an der seit 2007 geltenden Einschränkung. Steuerpflichtige können ihre Aufwendungen vorerst auch wieder nach der alten Rechtslage vor 2007 mit bis zu 1250 Euro geltend machen. Dazu muss ein Antrag auf Aussetzung der Vollziehung gestellt werden, der in der Regel gewährt wird, wenn der Einspruch gegen die Ablehnung eines Antrags auf Lohnsteuerermäßigung für die Jahre ab 2009, die Festsetzung von Einkommensteuervorauszahlungen für Veranlagungszeiträume ab 2009 oder Einkommensteuerbescheide $a b 2007$ gerichtet ist. 